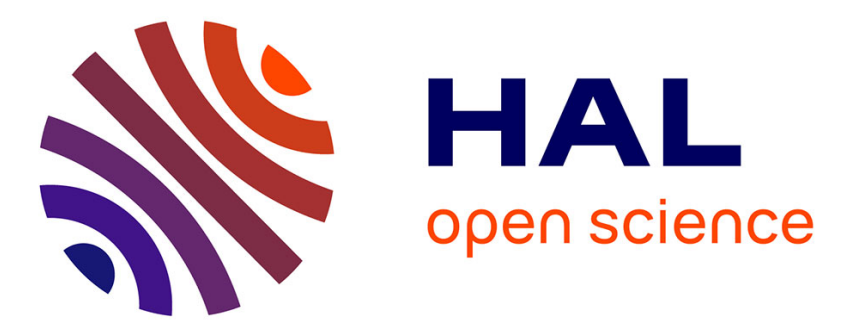

\title{
Toward a complete system for surveillance of the whole EEZ: ScanMaris and associated projects
}

Florent Jangal, Jean-Pierre Georgé, Alain Bonnot, Marie-Annick Giraud, Michel Morel, Aldo Napoli, Anne Littaye

\section{- To cite this version:}

Florent Jangal, Jean-Pierre Georgé, Alain Bonnot, Marie-Annick Giraud, Michel Morel, et al.. Toward a complete system for surveillance of the whole EEZ: ScanMaris and associated projects. OCEANS 2009, Oct 2009, Biloxi, United States. 4 p. - Article number 5422120 - ISBN: 978-142444960-6. hal-00569315

\section{HAL Id: hal-00569315}

https://hal-mines-paristech.archives-ouvertes.fr/hal-00569315

Submitted on 26 Jul 2013

HAL is a multi-disciplinary open access archive for the deposit and dissemination of scientific research documents, whether they are published or not. The documents may come from teaching and research institutions in France or abroad, or from public or private research centers.
L'archive ouverte pluridisciplinaire HAL, est destinée au dépôt et à la diffusion de documents scientifiques de niveau recherche, publiés ou non, émanant des établissements d'enseignement et de recherche français ou étrangers, des laboratoires publics ou privés. 


\title{
Toward a complete system for surveillance of the whole EEZ: ScanMaris and associated projects
}

\author{
F. Jangal ${ }^{1 \#}$, Member, IEEE, J.P. Georgé ${ }^{2}$, A. Bonnot ${ }^{3}$, M.A. Giraud ${ }^{4}$, M. Morel $^{3}$, A. Napoli ${ }^{5}$, A. Littaye ${ }^{6}$

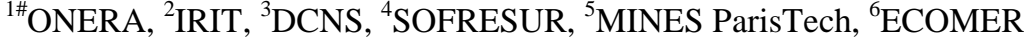 \\ http://www.irit.fr/scanmaris/ \\ florent.jangal@onera.fr
}

\begin{abstract}
There is currently an undeniable increase of maritime goods exchanges. As a consequence, maritime threats and risks are also rising. Innovative solution has to be developed to improve the security of this huge economic activity. Future generation of maritime surveillance system should allow: permanent and all weather coverage of maritime border areas, continuous collection of heterogeneous data provided by various sources, automatic detection of abnormal vessel behaviors, understanding of suspicious events, and early identification of threats. No equipment and information system deployments are at present able to answer all these requirements. We propose here an integrated system with relevant innovative technologies and capacities. The integrated system includes existing conventional and innovative sensors networks as well as new functionalities to track vessel movements and activities or detect abnormal vessel behaviors. The proposed high level engineering architecture is able to generate documented alarms using abnormal events. Those events are extracted from our intelligent maritime traffic picture. Thus, we aim to validate an end to end surveillance chain for future operational sea border surveillance.
\end{abstract}

\section{INTRODUCTION}

Overseeing vessel activities in the Exclusive Economic Zone (EEZ) is a technical challenge because of the huge area to monitor and the various activities to analyze. The first issue which crosses everyone's mind is: "how to detect vessels up to the EEZ limits?" High Frequency Surface Wave Radars (HFSWRs) can clearly overcome this issue [1]. Nevertheless, since radars, even completed by tracking or identification, cannot interpret vessels behavior, we propose a more cognitive approach [2].

The ScanMaris project, supported by the French National Research Agency, intends to develop a software workshop which continuously analyzes and marks abnormal vessel behavior. The project is structured in three processing layers supplying: a survey of global traffic using several sensors (traffic layer), an intelligent traffic map with history of vessel positions as well as various auxiliary information like vessel type/characteristics, maritime zoning or meteorological parameters (intelligent map layer), and an normality/abnormality status for each vessel (abnormal behavior layer). Identification of threats and constitution of investigation files are perform in the TAMARIS project.

ScanMaris started in January 2008 and we will be ready for demonstration end 2009. We present here current results

Authors want to warmly thank the French National Research Agency (ANR) for funding the ScanMaris Project and all steering committee members. dealing with sensor deployment, non-radar data collection and Adaptive Multi-Agent System (AMAS) for detection of abnormal behavior.

\section{SCANMARIS ARCHITECTURE}

A. End User Needs

Several discussions with the end users have permit to accurately defined ScanMaris outlines. These exchanges have also been useful to identify the succession of events which could describe abnormal behaviors.

Final project architecture and rules to be implemented in order to trig an alert have been derived from the analysis of end users needs.

\section{B. General Architecture}

The key element of the project is the enriched map (EM) which contains all information about vessels (i.e. position, insurance, class, route, etc.) and about scene layout (i.e. weather, registered maritime areas, bathymetry, etc.).

As shown in Fig. 1, all project elements are linked with EM. EM is a dynamic space-time object. Indeed, all events leaved on the map (e.g. new point of a vessel trail) have a position, a date, an identifier and a validity duration. The map must manage itself the evolution of events (e.g. decreasing of significance, storage, etc.). EM has also to select the relevant information to be sent to other compounds (e.g. vessels in vicinity, past anomaly, etc.)

1) Environmental Data Management: data dealing with bathymetry, weather, etc. are gathered by this module. If significant modifications of current data state occur, updated information is sent to the EM.

2) External Information Database: insurance or vessel history information is collected here. The main databases used by this module are the LLOYD insurance database and the TF2000 vessel database.

3) Old Event Storage: this is the "mass storage" of the project. All space-time events (e.g. vessel trail plus history of alert) can be stored in this module when they are no longer enough relevant to be kept in the EM.

4) End User: all the EM events are available to the end user. Tree level of alert can be sent to him: vigilance, pre-alert and alert. The user has the possibility to indicate to the system if an alert is unjustified or if the system has missed an abnormal behavior.

5) Inference Engine (IE): IE states either the normality or the abnormality of events. IE will not analyze the entire enriched map: this would be needlessly time consuming since 
fraud is a marginal activity. IE only answers to the requests send by Adaptive Multi-Agent Systems (AMAS) which extract relevant details.

6) Adaptive Multi Agent System: AMAS is the cognitive part of the project. Each vessel is associated to an agent. Each agent analyses its environment and significant modification of its environment highlights by the EM. Each agent leaves traces containing its current state and get traces of vessels in its vicinity area which contain current and former state of other agents. Dealing with this information, AMAS extract unusual event and request the analysis of inference engine.

7) Sensor database management: several sensors are used in the project. Some of them like the long range sensor, which is a High Frequency Surface Wave Radar (HFSWR), and the Automatic Identification System (AIS) receivers are operated by consortium member. Only data of other sensors are available (e.g. Vessel Monitoring System or VMS database).

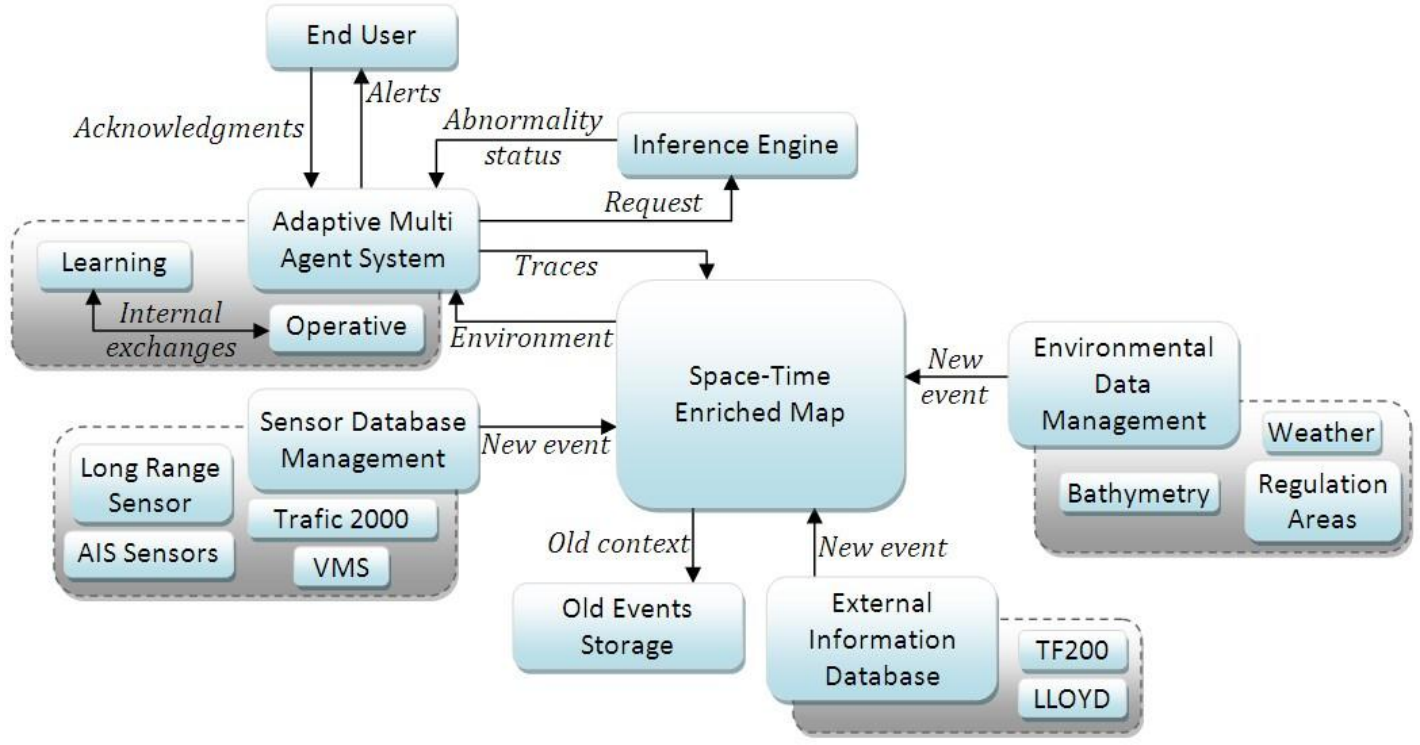

Fig. 1. Project architecture.

\section{SENSOR DATA}

As mentioned before, HFSWRs can detect targets up to the EEZ limit. However, for short range detection (i.e. few dozens of kilometers) other detection means are more suitable. Thus, it is useful to complement our HFSWR by cooperative beacons as Automatic Identification System (AIS) or Vessel Monitoring System (VMS) data.

Data obtained with all sensors (Fig. 2.) form the input of the ScanMaris workshop.
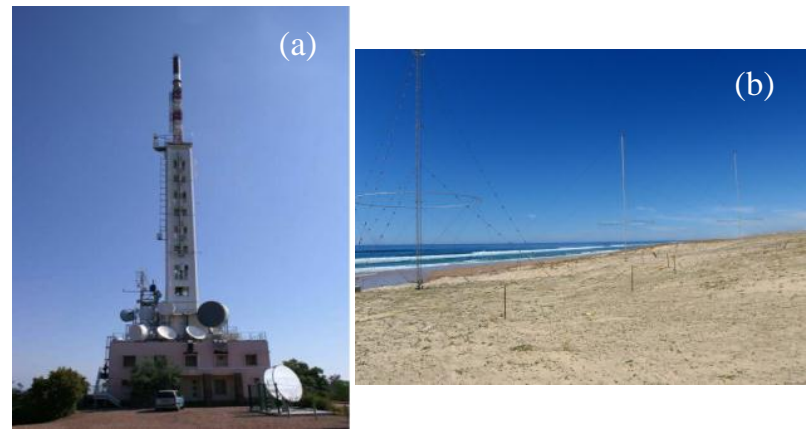

Fig. 2. Sensor sample: (a) AIS receiver, (b) HFSWR transmitting array.

Within the scope of the ScanMaris project sensors are not collocated. Collocated platforms will be deployed during 2010 in the south of France (in the frame of SisMaris project). Nevertheless, the data compatibility of all sensors has been tried and tested.
We are currently operating several sensors. Fig. 3. (a) shows a traffic map of the traffic separation scheme of Ushant as it is displayed on the ScanMaris interactive screen.
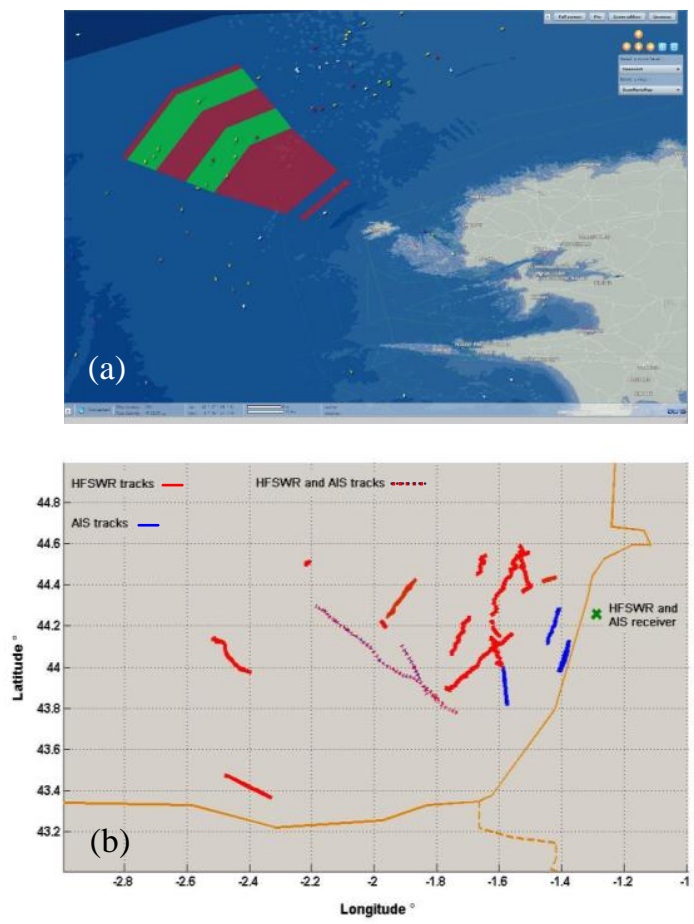

Fig. 3. Traffic map: (a) ScanMaris display unit, (b) HSFWR tracking and AIS information. 


\section{NON-SENSED DATA}

A key point of our approach is the widening of the data diversity to be analyzed.

One of the main constraints on the warehouses is the need of permanent accessibility and continuous update, despite the fact that stored elements have variable validity periods.

\section{A. Databases relating to vessels}

After a thorough survey of available databases, we have chosen pertinent ones and started to collect information (Fig. 4).

Data from different vessel related databases (Lloyds, Paris MOU, Fleet register, etc.) have been retrieved and fused in the ScanMaris vessel database. This additional information is used to enrich the dynamic traffic picture but the ScanMaris database can be directly queried by the cognitive elements (i.e. AMAS and inference engine) in order to state on the behavior abnormality. Moreover, the end user, if need be (e.g. research of information on doubtful vessels), could access to detailed data.

\section{B. Geographical server}

Geographical data have a particular handling [3]. Indeed, acquired data (e.g. NOAA, French oceanographic services, etc.) have to be reformatted, modeled, interpolated, structured in a database and made accessible via a web server. For example, the S57 standard of the International Hydrographic Organization is convenient for data exchanges but need to be carefully prepared before exploitation by a geographic information system.

We have chosen the ArcGIS software to manipulate the data [4]. A PostGIS database has been developed for the server since it is well suited for web queries [5].

Weather forecast, bathymetry, and S57 data are being integrated in the warehouse at the moment.

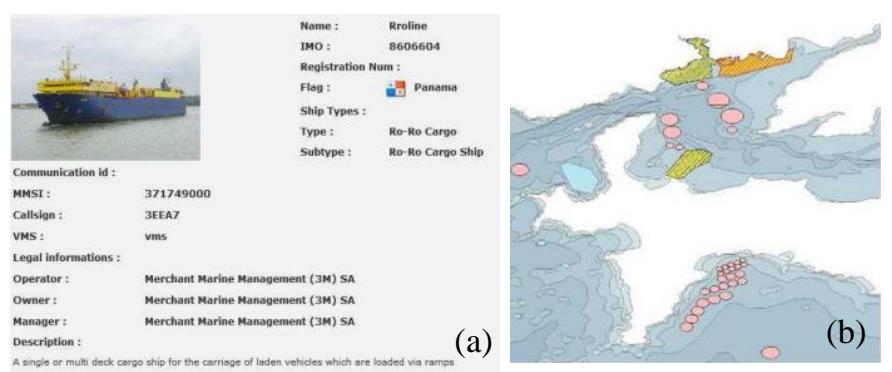

Fig. 4. (a) quasi-static information from ScanMaris database, (b) bathymetry with commercial ports (orange), naval ports (yellow), anchorage posts (pink), anchorage areas (blue).

\section{Cognitive ElEMENTS}

The AMAS can be thought as intelligent elementary entities (called agents) which have the ability to self-organize [6]. Thus, the objective or the global function to be achieved is spread over all agents and no centralized control is needed. In our case, each agent is a vessel. An agent exchanges information with others and with the enriched map.

After a training period, the self-organization of the agents (e.g. internal competences and global interactions) results in the ability to relevantly detect abnormal behaviors. AMAS are helped in this task by the rules engine which confronts basic facts (speed, type of vessel...) to sets of rules (legal, known simple behaviors...).

As shown in Fig.5, suspicious events increase the criticality level of an agent as it intelligently combines all the events happening to a vessel. According to the ratio between the forgetting lapse and event rate, the threshold can be overshot. In this case an alert is sent to the user.

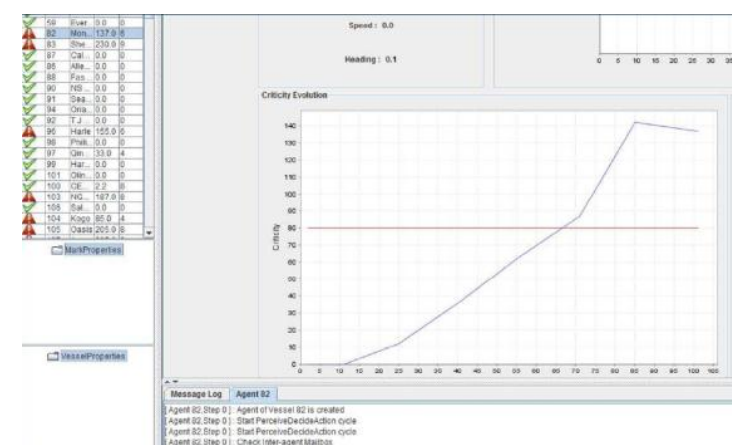

Fig. 5. Extract of the AMAS management GUI. Left: list of agents with state (normal or alert). Right: evolution of the criticity of an agent (threshold in red).

\section{PERSPECTIVES}

Global surveillance of the Exclusive Economic Zone is an international concern for years. Active and autonomous surveillance of the whole EEZ is the objective of our research actions.

We aim to propose a complete monitoring system which is able to continuously detect vessels, synthesize an intelligent traffic map (vessels position, insurance information, sea state, etc.), point out abnormal behavior of vessels, interpret suspicious activities and identify potential threats.

ScanMaris encompasses the first part of the proposed approach. It will be completed by the TAMARIS project (also partly financed by the French Research Agency) which has just begun. TAMARIS outputs can be directly addressed to the legal authorities as first elements of an investigation file.

ScanMaris is still in progress. Demonstration phase will start in the few coming months and the final results will be presented at the conference. We will also present some progress on the TAMARIS project.

\section{REFERENCES}

[1] M. Menelle, G. Auffray, F. Jangal, "Full digital High Frequency Surface Wave Radar: French trials in the biscay bay", in Proc. Radar 2008 Conf., pp. 224-229, DOI 10.1109/RADAR.2008.4653922.

[2] M. Morel et al, "Developing and Evaluating Solutions of Prevention and Monitoring Maritime Borders: ScanMaris", in Proc. MAST 2007 Conf., Genoa, Italy.

[3] J. Iris, J. Chemitte, A. Napoli, “The geophysical web - part 5", Springer London, 2007, DOI 10.1007/978-1-84628-827-2_17

[4] ESRI, "ArcGIS engine", http://www.esri.com/software/arcgis/ arcgisengine/index.html

[5] Refractions Research Inc, "PostGIS", http://postgis.refractions.net/ 
[6] M.-P. Gleizes, V. Camps, J.-P. Georgé, and D. Capera. "Engineering Systems which Generate Emergent Functionalities". Springer Berlin, 2008, DOI 10.1007/978-3-540-85029-8_5

[7] M. Morel et al, "TAMARIS", in Proc. MAST 2009 Conf., Kista, Sweden. 